\title{
Knowledge and Attitude of Dentists toward Silver Diamine Fluoride in Saudi Arabia
}

\author{
Abdullah Faraj Alshammari ${ }^{1}$, Rana Homoud Alenzi ${ }^{2}$, Ashwaq Abdullah Alanezi ${ }^{3}$, Abdulmjeed Sadoon Enizy ${ }^{4}$, \\ Ahmed Mohmmed Aldakhil ${ }^{5}$, Khlood Abdulkader Alkurdi ${ }^{6}$
}

\begin{abstract}
Introduction: Dental caries is managed by two approaches: prevention which focuses on inhibiting the caries progression, and the traditional approach which is treating caries by drilling and filling. Logically, the first approach is more effective. One of the recently marketed preventive methods for preventing and inhibiting the progression of dental caries is silver diamine fluoride (SDF), which is proven to be minimally invasive and cost-effective, SDF has proven to have a significant success rate in caries prevention, especially in the early childhood caries.

Objective: The study aims to evaluate general practitioners'SDF clinical experience, knowledge, professional behavior, and attitudes.

Materials and methods: The study was a cross-sectional study and we utilized a random sampling of Saudi general dental practitioners from different places in the Hail region, KSA. The study was conducted using papered interview and an online questionnaire followed by educating the dentists about SDF.

Results: Dentists were asked about SDF, $62.7 \%$ answered that they heard about SDF topical application, but the dentists who answered all questions correctly are only $14.89 \%$ with no statistically significant difference among gender, age, or sector.

Conclusion: This study had several limitations. It only included the dental GPA in the Hail region, Saudi Arabia. As is the case with all surveys, only a limited number of questions could be asked. Thus, the information to be obtained is also limited. Future research should therefore include other questions to clarify other aspects which we could not explore in this research.

Keywords: Arrested caries, Caries, Pediatric dentist, Prevention, Primary dentition, Silver diamine fluoride.

International Journal of Clinical Pediatric Dentistry (2021): 10.5005/jp-journals-10005-2009
\end{abstract}

\section{INTRODUCTION}

Dental caries is considered one of the most prevalent chronic dental diseases. ${ }^{1}$ It affects all social classes in society. However, caries risk is considered a variable within communities, and there are low-risk and high-risk communities. ${ }^{2}$ Many factors that control the level of caries risk, including diet, socioeconomic status, demographic factors, and level of educations. ${ }^{3}$ Dental caries is managed by two approaches: prevention which focuses on inhibiting caries progression, and the traditional approach which is treating caries by drilling and filling. Logically, the first approach is more effective. One of the recently marketed preventive methods for preventing and inhibiting the progression of dental caries is silver diamine fluoride (SDF), which is proven to be, minimally invasive and costeffective. ${ }^{4-6}$ Silver diamine fluoride has proven to have a significant success rate in caries prevention, especially in early childhood caries. $^{7-9}$ It gives an alternative choice for the management of dental caries, especially if caries is at its early stages, without pulpal signs and symptoms. Also, it could save time and effort for the dental team and families. It is also considered to be a harmless approach for uncooperative patients. Thus, it could prevent any psychological and emotional impacts, that might happen as an outcome of more aggressive treatment options. On the contrary, SDF has its own disadvantages. One of these is staining. It stains the enamel and dentin with a dark stain, which might cause some esthetic concerns. ${ }^{10}$ The application of SDF once yearly has been proven to be slightly less effective than twice yearly. ${ }^{11}$ When applied twice yearly, the $38 \%$ SDF solution has a success rate of $84.8 \%$ for stopping the progression of dental caries. ${ }^{10}$ Furthermore, the $38 \%$ SDF was shown to be more effective than the $12 \%$ SDF $^{6,12}$ the study aimed to evaluate SDF clinical experience, knowledge, professional
${ }^{1}$ Department of Basic Dental and Medical Science, College of Dentistry, University of Hail, Hail, Kingdom of Saudi Arabia

${ }^{2}$ King Abdulaziz Medical City, Riyadh, Kingdom of Saudi Arabia

${ }^{3}$ Ministry of Health, Najran, Kingdom of Saudi Arabia

${ }^{4}$ Department of Dentistry, Prince Sultan Military Medical City, Riyadh, Kingdom of Saudi Arabia

${ }^{5}$ Dental Research Center, College of Dentistry, Qassim University, Buraidah, Kingdom of Saudi Arabia

${ }^{6}$ Barts and the London School of Medicine and Dentistry, Queen Mary University of London, London, UK

Corresponding Author: Abdullah Faraj Alshammari, Department of Basic Dental and Medical Science, College of Dentistry, University of Hail, Hail, Kingdom of Saudi Arabia, Phone: +966543334774 , e-mail: abdullahfs5@gmail.com

How to cite this article: Alshammari AF, Alenzi RH, Alanezi AA, et al. Knowledge and Attitude of Dentists toward Silver Diamine Fluoride in Saudi Arabia. Int J Clin Pediatr Dent 2021;14(5):662-665.

Source of support: Nil

Conflict of interest: None

behavior, and attitudes of dental general practitioners (GPs), in the Hail region, Saudi Arabia.

\section{Materials and Methods}

The study was a cross-sectional study and we utilized a random sampling of Saudi general dental practitioners from different places in the Hail region, KSA. The team carried three dentists, the data were conducted using a papered interview, and an online questionnaire 
followed by educating the dentists about SDF (advantages, disadvantages, indications, and contraindications). The period for conducting and assessing was from 28-4-2019 to 25-5-2019. Each general dentist was asked to sign a written ethical consent during the questionnaire's interview. The informed ethical consent form was designed and approved by the Ethical Committee of the University of Hail, KSA. The study aimed to evaluate GPs' SDF clinical experience, knowledge, professional behavior, and attitudes, in the Hail region, Saudi Arabia. The survey was distributed among 150 Saudi general dental practitioners, consisted the demographic data such as age, gender, clinical experience, sector, and BDS university followed by informed consent to authorize us to publish the data. The questions included were 11, with a multiple-choice style. The first question was that if they have heard about SDF or not, if they did, they should continue answering the remaining questions. The 2nd, 3rd, and 9th questions were about the advantages and disadvantages of SDF, 4th and 5th questions were related to the main indications and contraindications of SDF, the 6th question was regarding the type of patients that SDF treatment is not suitable for them, the 7th and 8th questions were to examine the GPs knowledge for the most effective way to use SDF regarding the frequency and intervals, the 10th question was about the informed consent and the last question was about GPs anticipation for any future implementation of SDF replacing the traditional methods.

\section{Results}

Our study was a cross-sectional study conducted using a pretested questionnaire in 180 dental practitioners. The response rate was $83.33 \%$. In the questionnaire, we mainly assessed the knowledge about the SDF topical application. When we measured that how many dentists answered the correct options for all the knowledgebased questions, we observed that 14 (14.89\%) dentists answered all the questions as correct. When the relationship of various sociodemographic details of dentists was measured with the knowledge of SDF, it was observed that age and gender did not have any statistically significant association with the knowledge of SDF among dentists $(p>0.05)$ (Table 1). There was no association observed with the sector that dentists working in, and the knowledge of SDF (Table 1).

When we assessed the relationship between various sociodemographic variables of dentists such as age, gender, and sector of practice with dentists who have answered all the knowledge questions as correct, it was found that there was no statistically significant association observed with any of the above variables ( $p>0.05$ ) (Fig. 1).

The first question asked was "Have you ever heard about silver diamine fluoride?" We observed that $62.7 \%(n=94)$ reported that they have heard of SDF (Fig. 2). Those who answered "yes" to the above question were asked to answer the remaining questions related to the SDF topical agent.

The next question was "Which of the following do you think is an advantage of using silver diamine fluoride?". For this, 55.3\% ( $n=52$ ) answered the correct answer (caries arresting) (Table 2).

When a question was asked about the main disadvantage of using SDF, $47.9 \%(n=45)$ answered the correct option (staining to the tooth). $27.7 \%$ answered it as "Irritation to pulp" whereas $14.9 \%$ of the dentist mentioned it as "low pH" (Table 3).

The next question was about the main indication of using SDF (Fig. 3). For this question, $51.1 \%(n=48)$ dentists answered the correct option "Cavitation of primary teeth". $27.7 \%$ mentioned it as "Cavitation to permanent teeth", 12.8\% mentioned it as "Incipient

Table 1: Relationship of sociodemographic details with knowledge of SDF

\begin{tabular}{|c|c|c|c|c|c|c|}
\hline \multirow{2}{*}{ Have you ever heard of SDF? } & \multicolumn{2}{|c|}{ Age } & \multicolumn{2}{|c|}{ Gender } & \multicolumn{2}{|c|}{ Sector } \\
\hline & $25-35$ & $36-45$ & Male & Female & Government & Private \\
\hline Yes & 93 & 1 & 59 & 35 & 73 & 21 \\
\hline No & 55 & 1 & 34 & 22 & 49 & 7 \\
\hline Total & 148 & 2 & 93 & 57 & 122 & 28 \\
\hline Chi-square value & 0.13 & & 0.627 & & 2.238 & \\
\hline$p$ value & 0.70 & & 0.802 & & 0.135 & \\
\hline
\end{tabular}

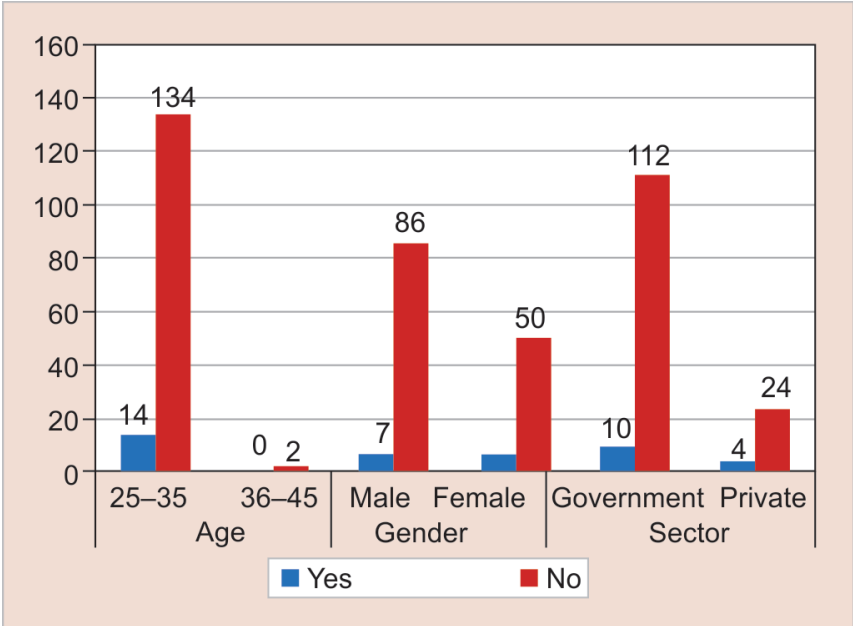

Fig. 1: Relationship of a sociodemographic variable with dentists who answered all of the questions correctly

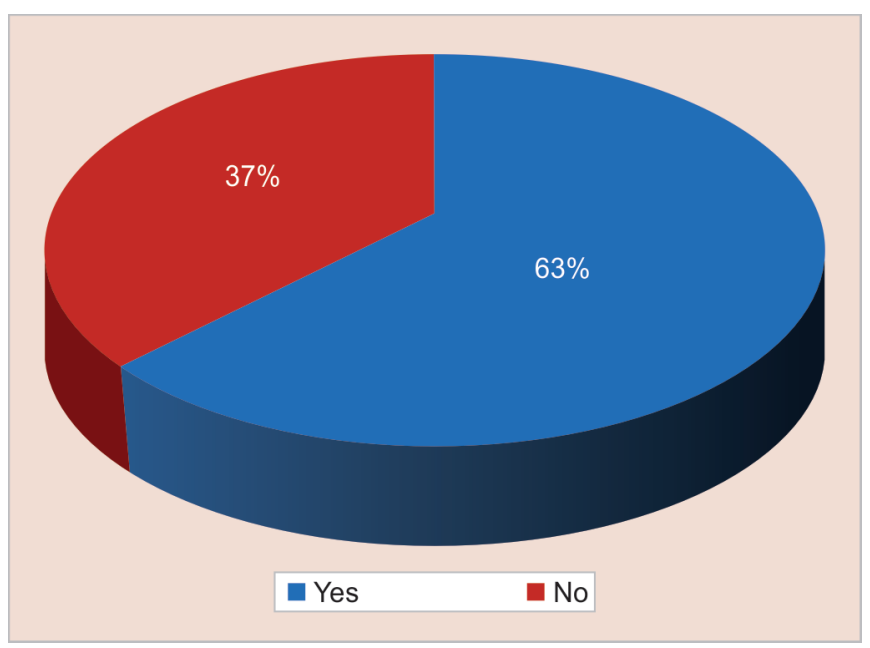

Fig. 2: Have you ever heard about silver diamine fluoride? 
Table 2: Which of the following do you think is an advantage of using silver diamine fluoride? (Q3)

\begin{tabular}{lllc}
\hline & & Frequency & Percent \\
\hline Options & Fluoride releasing & 26 & 27.7 \\
& Caries arresting & 52 & 55.3 \\
& Esthetically pleasing & 12 & 12.8 \\
Mummification & 4 & 4.3 \\
& Total & 94 & 100.0 \\
\hline
\end{tabular}

Table 3: Which of the following do you think is a disadvantage of SDF? (Q4)

\begin{tabular}{lllc}
\hline & & Frequency & Percent \\
\hline Options & Staining to the tooth & 45 & 47.9 \\
& Irritation to pulp & 26 & 27.7 \\
& Low pH & 14 & 14.9 \\
Toxicity & 9 & 9.6 \\
Total & 94 & 100.0 \\
\hline
\end{tabular}

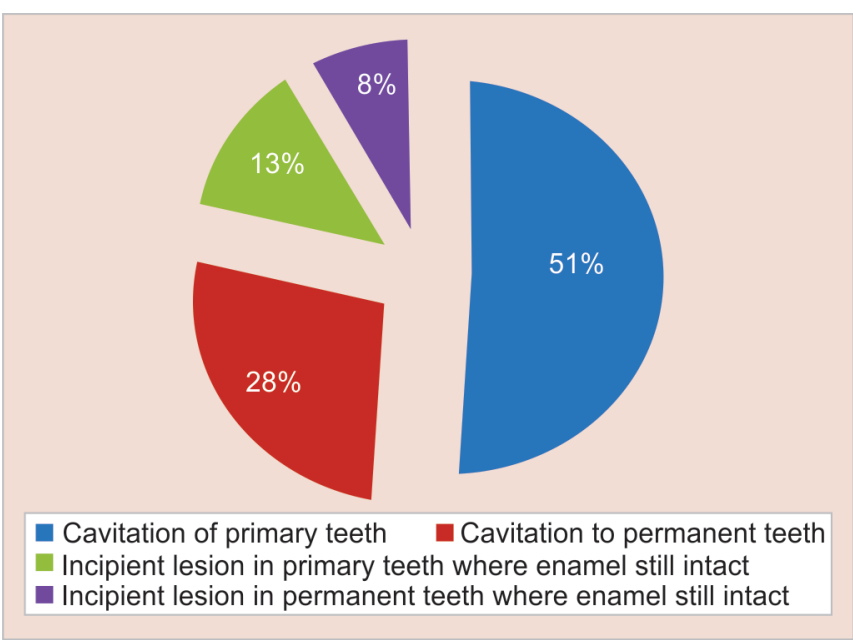

Fig. 3: Indications of SDF as answered by the participants

Table 4: Which of the following do you think is the main contraindication of SDF?

\begin{tabular}{lllc}
\hline & & Frequency & Percent \\
\hline Options & Symptomatic pulp & 47 & 50.0 \\
& inflammation & & \\
& Dentin caries & 11 & 11.7 \\
& Enamel caries & 7 & 7.4 \\
& Root caries & 29 & 30.9 \\
& Total & 94 & 100.0 \\
\hline
\end{tabular}

lesion in primary teeth where enamel still intact", and the remaining $8.5 \%$ answered it as "Incipient lesion in permanent teeth where enamel still intact".

When a question regarding the contraindication of SDF was asked, 50\% ( $n=47)$ mentioned the correct answer ("Symptomatic pulp inflammation"). Other dentists answered it as "Dentin caries" (11.7\%), "enamel caries" (7.4\%), and root caries (30.9\%) (Table 4).

Most of the dentists (66\%) answered the correct option "Allergy to SDF component" to the question "Which of the following groups do you think is not a suitable candidate for SDF?" (Table 5).
Table 5: Which of the following groups do you think is not a suitable candidate for SDF?

\begin{tabular}{lllc}
\hline & Frequency & Percent \\
\hline Options & $\begin{array}{l}\text { Medically compro- } \\
\text { mised patient }\end{array}$ & 18 & 19.1 \\
& $\begin{array}{l}\text { Allergy to SDF compo- } \\
\text { nent }\end{array}$ & 62 & 66.0 \\
& Autistic patient & 10 & 10.6 \\
& $<4$-year-old patient & 4 & 4.3 \\
& Total & 94 & 100.0 \\
\hline
\end{tabular}

Table 6: How many applications do you think that SDF needs to be effective?

\begin{tabular}{lllc}
\hline & & Frequency & Percent \\
\hline Options & One & 35 & 37.2 \\
& Two & 42 & 44.7 \\
& Three & 17 & 18.1 \\
& Total & 94 & 100.0 \\
\hline
\end{tabular}

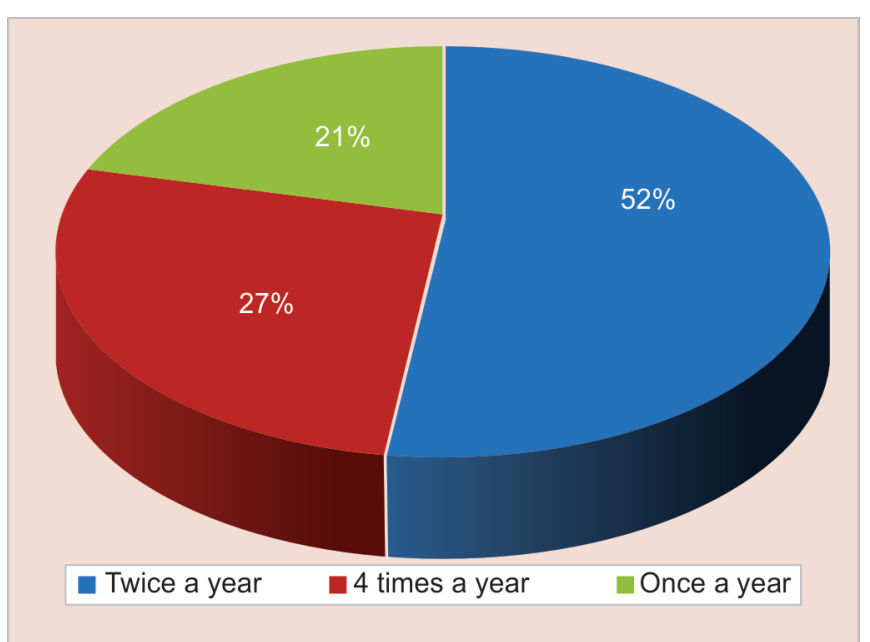

Fig. 4: Intervals of application of SDF as answered by participants

44.7\% ( $n=42)$ said that "two applications" of SDF should be done to give its effect maximum. Whereas $37.2 \%$ said it as "one application" would be effective (Table 6).

For the question regarding the interval of application of SDF in a year, $52.1 \%(n=49)$ mentioned this as "twice a year", which is the recommended interval of application. Some (26.6\%) mentioned this as "4 times a year" (Fig. 4).

When questions regarding staining to teeth by the use of SDF was asked, $31.9 \%(n=30)$ dentists answered it as "Staining to teeth is permanent, while to the skin is temporary" which is an incorrect statement. Only $14.9 \%(n=14)$ answered the correct statement "Staining to teeth is temporary, while to the skin is permanent". $29.8 \%$ answered it as "Staining to teeth and skin is permanent" (Fig. 5).

Most of the participants (74.5\%) agreed that they should take consent from parents before applying SDF, whereas $25.5 \%$ said that it is not necessary (Table 7).

Seventy-two percent of dentists believe that SDF will be their favorite fluoride application option in the future rather than the traditional ones (Fig. 6). 


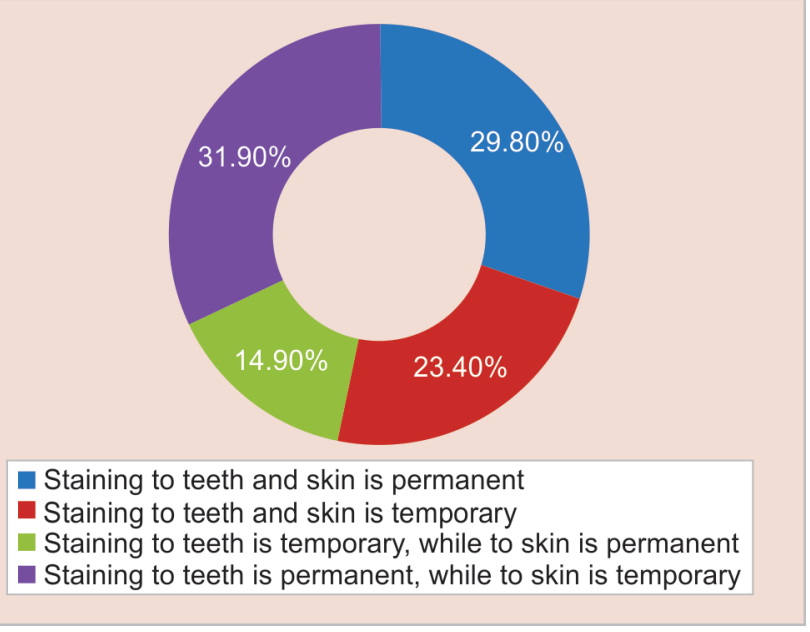

Fig. 5: Disadvantages of SDF as reported by the participants

Table 7: Do you think that it is important to take a consent form from the parents for the application of SDF?

\begin{tabular}{lllc}
\hline & & Frequency & Percent \\
\hline Options & Yes & 70 & 74.5 \\
& No & 24 & 25.5 \\
& Total & 94 & 100.0 \\
\hline
\end{tabular}

\section{Discussion}

The surveys were delivered using a papered interview and an online questionnaire, thus the response rate was high (83.33\%), followed by educating the dentists about SDF (advantages, disadvantages, indications, and contraindications). Regarding SDF education, $62.7 \%$ answered that they heard about SDF topical application, but the dentists who answered all questions correctly are only $14.89 \%$ with no statically significant difference among gender, age, or sector. This finding is not surprising because SDF had not been cleared by the FDA until 2014, and in this country, Saudi Arabia, where we are doing our survey, SDF is not cleared yet till this year (2019) by Saudi Food and Drug Authority (SFDA).

We have noticed that when the participants were asked about the main advantage, disadvantages, indications, and contraindications of SDF, that there was a similarity in the correct answers' low percentages. This only indicates that there is a lack of knowledge even among the dentists who said that they have heard about SDF. The clinical application of SDF is advised to be applied two times in 1 year for a better outcome, although that would increase the likelihood of discoloration. The discoloration is the main concern and disadvantage of this material, while teeth discoloration is permanent, discolored skin is temporary but that does not mean that dentists should not be careful and take the required precaution while applying SDF. This information was the least to be known among the dentists, only 31.9\%. Consent from the parents is a must, for medicolegal purposes, and out of the 94 dentists, 70 agreed on this. When we discussed the correct answers with the participants, $72 \%$ of them showed us that they are very interested in this material, and they are planning to use it in the future. This study had several limitations. It only included the dental GPs in the Hail region, Saudi Arabia. As is the case with all

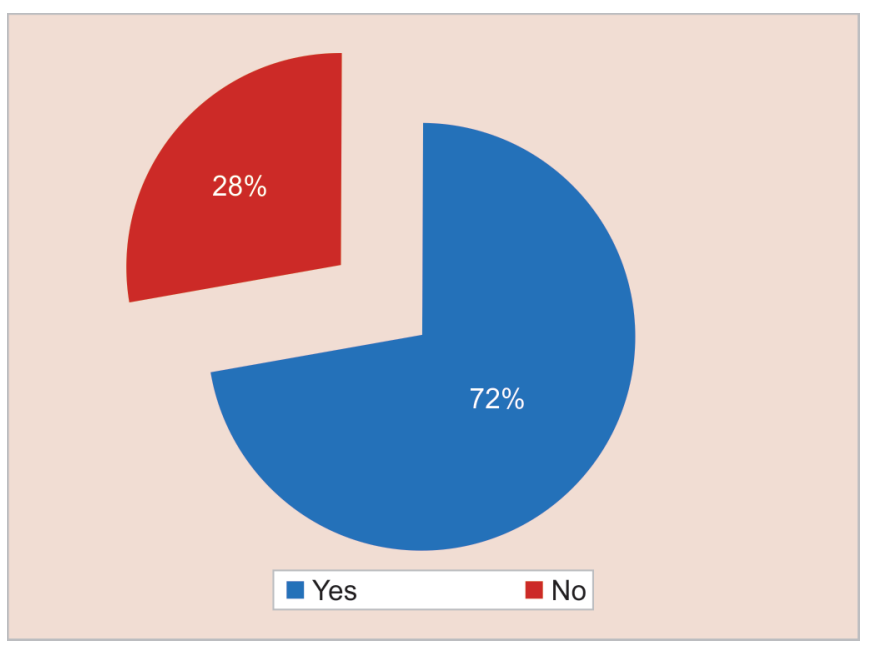

Fig. 6: Do you think SDF will be your favorite in the future rather than the traditional method?

surveys, only a limited number of questions could be asked. Thus, the information to be obtained is also limited. Future research should therefore include other questions to clarify other aspects which we could not explore in this research.

\section{References}

1. Dülgergil Ç, Dalli M, Hamidi M, et al. Early childhood caries update: a review of causes, diagnoses, and treatments. J Nat Sci, Biol Med 2013;4(1):29. DOI: 10.4103/0976-9668.107257.

2. Peker I, Mangal T, Erten $\mathrm{H}$, et al. Evaluation of caries risk in a young adult population using a computer-based risk assessment model (Cariogram). J Dent Sci 2012;7(2):99-104. DOI: 10.1016/j. jds.2012.03.004.

3. Costa SM, Martins CC, Bonfim MLC, et al. A systematic review of socioeconomic indicators and dental caries in adults. Int J Environ Res Public Health 2012;9(10):3540-3574. DOI: 10.3390/ijerph9103540.

4. Chu CH, Lo ECM. Promoting caries arrest in children with silver diamine fluoride: a review. Oral Health Prev Dent 2008;6(3):15-21.

5. Peng J, Botelho M, Matinlinna J. Silver compounds used in dentistry for caries management: a review. J Dentis 2012;40(7):531-541. DOI: 10.1016/j.jdent.2012.03.009.

6. Yee R, Holmgren C, Mulder J, et al. Efficacy of silver diamine fluoride for arresting caries treatment. J Dent Res 2009;88(7):644-647. DOI: 10.1177/0022034509338671.

7. Mock C, Donkor P, Gawande A, et al. Essential surgery: key messages from disease control priorities. 3rd ed. The Lancet 2015;385(9983):2209-2219. DOI: 10.1016/S0140-6736(15)60091-5.

8. Sharma G, Puranik MP. Approaches to arresting dental caries: an update. J Clin Diagnos Res: JCDR 2015;9(5):ZE08. DOI: 10.7860/ JCDR/2015/12774.5943.

9. Mei ML, Chin-Man Lo E, Chu C-H. Clinical use of silver diamine fluoride in dental treatment. Compend Contin Educ Dent 2016;37(2):93-98.

10. Llodra JC, Rodriguez A, Ferrer B, et al. Efficacy of silver diamine fluoride for caries reduction in primary teeth and first permanent molars of schoolchildren: 36-month clinical trial. J Dent Res 2005;84(8):721-724. DOI: $10.1177 / 154405910508400807$.

11. Zhi Q, Lo E, Lin H. Randomized clinical trial on effectiveness of silver diamine fluoride and glass ionomer in arresting dentine caries in preschool children. J Dentis 2012;40(11):962-967. DOI: 10.1016/j. jdent.2012.08.002.

12. Fung $M$, Duangthip $D$, Wong $M$, et al. Randomized clinical trial of $12 \%$ and 38\% silver diamine fluoride treatment. J Dent Res 2017;97(2):171178. DOI: $10.1177 / 0022034517728496$. 